\title{
Meal Data Sponsor-Defined Identifier
}

National Cancer Institute

\section{Source}

National Cancer Institute. Meal Data Sponsor-Defined Identifier. NCI Thesaurus. Code C162138.

One or more sponsor defined characters used to identify, name, or characterize the meal data. 